\title{
Design and Implementation of Three-phase Electric Parameter Acquisition Module Based on STM32F103 and ATT7022E in Intelligent Street Light Control System
}

\author{
Yimin Nan \\ School of Airport Management of Guangzhou Civil Aviation College \\ Guangzhou Guangdong 510403, China \\ submitfile@163.com
}

Keywords: intelligent street light control system; electric power parameter; STM32F103; ATT7022E; power factor

Abstract. To meet the need of electric power parameter acquisition in intelligent street light control system, STM32F103 as main control chip, ATT7022E for electric energy metering chip, three-phase power parameter acquisition module is designed. This module has the instantaneous voltage and current, active and reactive power, power factor and so on electric parameter acquisition, storage and display function, also uploads data to host computer through the 485 bus.

\section{Introduction}

As China's urbanization speeding up, the urban road lighting has become increasingly important. As an important part of urban infrastructure, the emergence of intelligent street light control system greatly ease the burden of the management and maintenance of lighting system, and from a certain extent, reduce the energy loss. Due to the vigorously policy support, together with a vast market, profit is relatively higher, street light control system gets fast development, scientific research institutes and the company of research street light control system also carried out extensive research of intelligent street light control system: Qufu Normal University, based on GPRS technology, this paper puts forward a kind of cross-regional centralized management intelligent street light control system design based on B/S structure ${ }^{[1]}$; College of Electronic Information and Automation, Tianjin University of Science \& Technology, using GPRS and ZigBee wireless communication technology, takes an AVR microcontroller (MCU ) as the control core to adjust the brightness of the LED lights automatically with the surrounding brightness detected in real time ${ }^{[2]}$; School of information engineering Wuyi university realized the cable monitoring, lamp fault positioning and intelligent monitoring through SENS-01 module and addressable technology ${ }^{[3]}$. In intelligent street light control system, collect power parameters (current, voltage, etc.) to facilitate the use and management of users, but for the electric parameter acquisition such as three-phase voltage, current effective value, each phase or the conjunction of active power, reactive power, apparent power, power factor, frequency, phase and other parameters testing, often use the integrated operational amplifier discrete electronic devices in the design of the traditional three phase, such a large and complex circuit, not only the cost is very high, and the design, debugging is likely to be encountered great difficulties, some functions even difficult to achieve. This paper puts forward three-phase electric parameter acquisition module based on ATT7022E energy metering chip can solve the above problem very well.

\section{Electric energy metering chip}

ATT7022 $\mathrm{E}^{[4-7]}$ production of hi-trend Technology (Shanghai) Limited, high precision, low power consumption, with zero line current, three-phase electric energy metering chip, suitable for three-phase three wire and three-phase four line application. The operating voltage is $3.3 \mathrm{~V}$, the dynamic range of the 5000:1 power measurement accuracy is better than $0.1 \%$, the standard SPI interface, the rate of up to $10 \mathrm{Mbps}$, and provides four flexible pulse output. Internal integration 7 channel 19 bit second-order sigma delta ADC, reference voltage circuit and digital signal processing circuit, ADC sampling rate up 
to $28 \mathrm{KHZ}$, able to measure the total (fundamental wave and harmonic), active power, reactive power, apparent power, and the phase current and voltage effective value, power factor, phase Angle, frequency, because of all the circuit parameters are internal algorithm directly to the result of the operation, so as to fully guarantee the accuracy and reliability of the parameters. In addition, the voltage angle precision can reach 0.1 degrees, and the refresh rate of all kinds of parameters can reach $28.8 \mathrm{~Hz}$. The use of digital filter mode, the normal mode of the power consumption of 7 ADC is $6.5 \mathrm{~mA}$, the total power loss is only $4 \mathrm{~mA}$. Also provide the current and voltage of the synchronous ADC data, the data can be stored in the $1 \mathrm{~K}$ bytes cache, greatly facilitate the application of harmonic analysis.

\section{The system design}

The design of the three-phase power parameter acquisition module mainly by ATT7022E, STM32F103 as main control chip, real time clock, FLASH storage, keyboard, LED display, RS485 bus. Hardware system block diagram is shown in Fig. 1.

ATT7022E is used to measure the parameters, three-phase voltage, three-phase current signals are $\mathrm{AC}$ voltage differential input mode, as long as the measured voltage and current through the voltage transformer, current transformer, voltage or current transformer step-down to chips allow range, by way of differential input chip, automatic measurement of various parameters can be achieved.

STM32F103 master control chip reads data from ATT7022E through the SPI interface, through 485 serial communicates with the host computer or other equipment, real-time clock provides precise time and date, FLASH is mainly used to store all kinds of electricity parameters, each period of electricity, electric energy meter constant, time parameters etc. an external EEPROM (AT24C1024) to store more data, the keyboard is used to input the meter checking data, set the alarm limit parameters, query operation data, the data show use LED.

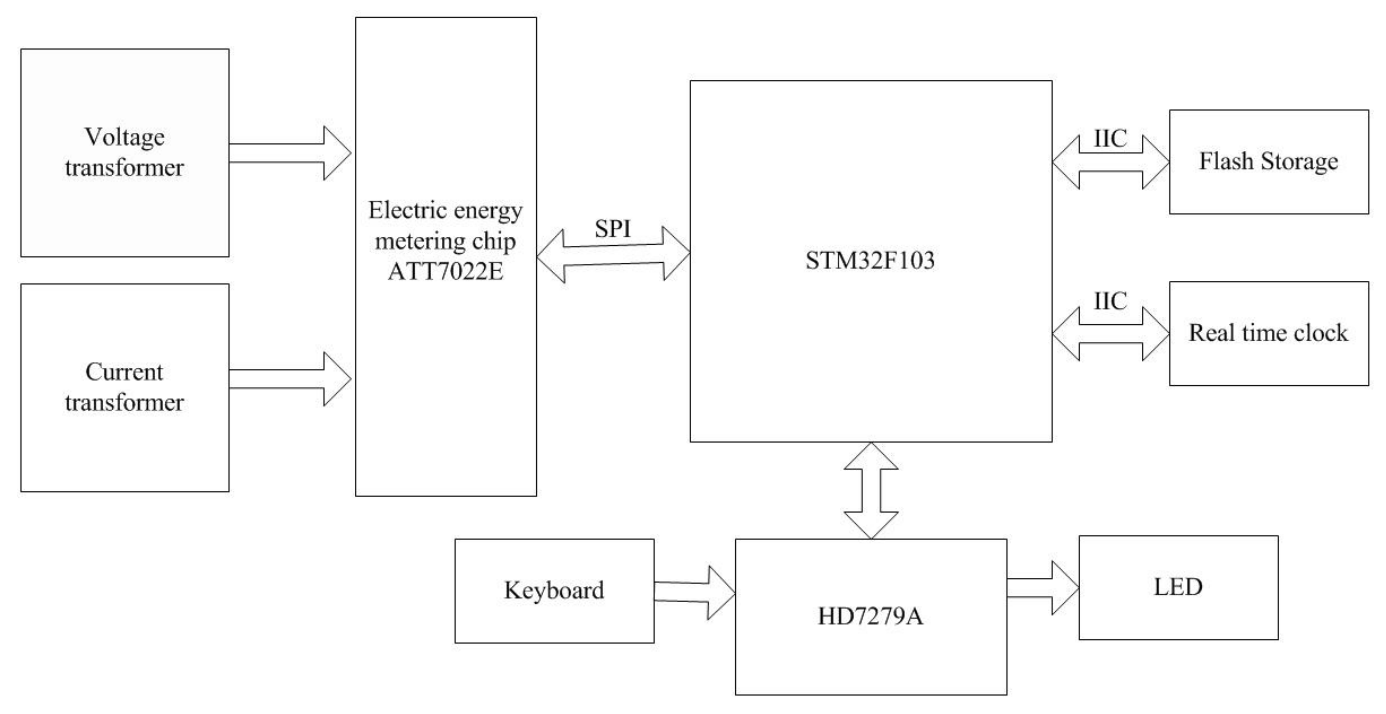

Fig. 1. Hardware structure

\section{The hardware circuit design}

\section{Master control chip selection}

The main control chip reads and writes registers of ATT7022E through the SPI interface, through the USART communities with the host computer and other devices, through the IIC interface to complete the data storage, so the requirements of the main control chip is very high. By comparing the internal resources and the cost of the chip, the STM32F103 chip based on CORTEX-M3 ARM core is selected.

\section{Analog input circuit}

ATT7022E integrated 7 channel 19 bit A/D converter, current effective value in the range of $0.1 \mathrm{mV}$ to $500 \mathrm{mV}$, the linear error is less than $0.1 \%$; the channel voltage effective value in the range of $0.2 \mathrm{mV}$ to 
$500 \mathrm{mV}$, the linear error is less than $0.1 \%$; the effective value of voltage is from $0.2 \mathrm{~V}$ to $0.5 \mathrm{~V}$, the current value of $0.2 \mathrm{mV}$ to $500 \mathrm{mV}$, the power of linear error is less than $0.1 \%$.

Three-phase meter measurement chip samples the voltage using voltage transformer, isolated chip and power grid, which can obtain good anti-interference performance.

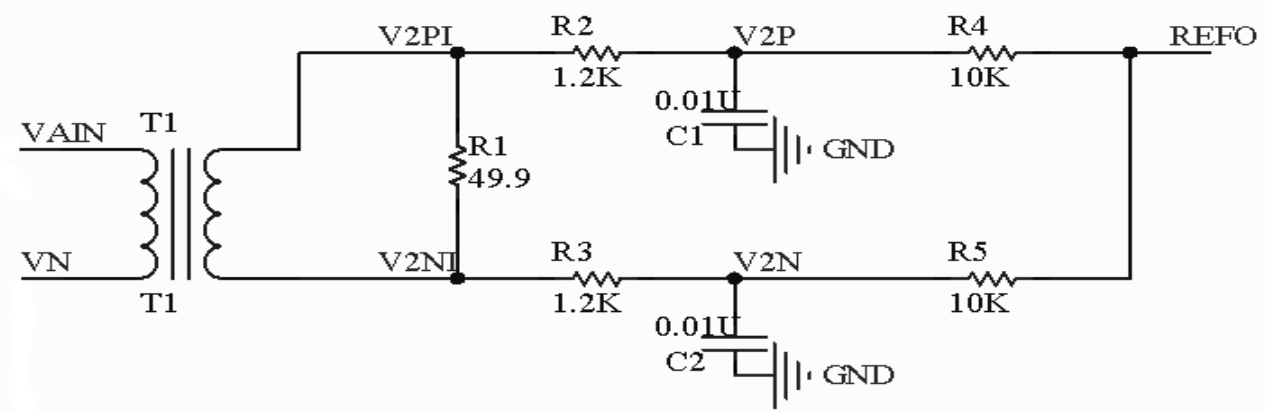

Fig. 2. Voltage sampling circuit

The sampling value on chip voltage input pin is $0.2 \sim 0.5 \mathrm{~V}$, so choose LCTV31CE micro precision voltage transformer $(2 \mathrm{~mA} / 2 \mathrm{~mA})$, as shown in Fig. 2, voltage sampling circuit adopts the differential input method. VAIN, VN for grid voltage, V2PI, V2NI for sampling the voltage input pin.

Current sampling is completed through the current transformer, this design uses 400/5A current transformer, two secondary coil and resistor of $49.9 \Omega / 10 \mathrm{~W}$, full of $400 \mathrm{~A}$ when the input voltage is $1 \mathrm{~V}$, IA1, IA2 in Fig. 3 for secondary side of Current transformer, V1P, V1N for chip sampling current input pin.

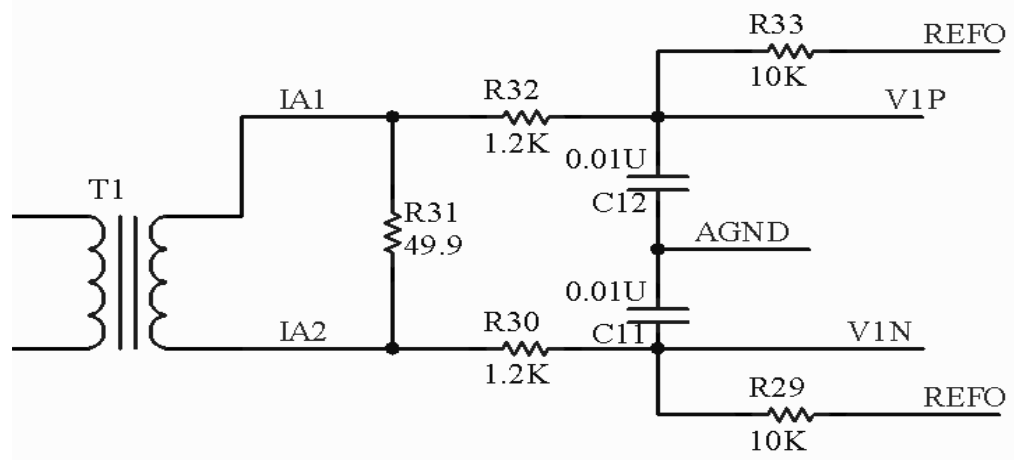

Fig. 3. Current sampling circuit

\section{Display and keyboard circuit}

In order to reduce costs, saving IO resources, enhance the reliability of the system, display and keyboard circuit adopts HD7279A chip, HD7279A is a piece with a serial interface smart display driver chip, can drive a total of 8 common cathode digital tube (or 64 LED), can also connect up to 64 keys matrix, the single chip can complete all the LED display, keyboard interface functions.

\section{Software design}

Because the ATT7022E can automatically complete the measurement of parameters, CPU only needs to operate the registers in electric energy metering chip, which greatly simplifies the software program. Software flow chart is shown in Fig. 4.

Initialization including I/O port of STM32F103, SPI, UART, keyboard display, measurement chip initialization. The meter checking is the compensation for the current gain, voltage gain, power gain and phase of each phase. Phase correction can be carried out according to the accuracy requirements, considering the subsection or not. Subsection is divided according to the magnitude of the current, ATT7022E can be divided into 2 sections for phase compensation. When software meter checking, in general, voltage, current calibration, starting current Settings, set threshold voltage, off phase threshold voltage settings, there is no requirement on the order. But in the power gain correction, please note that first set the conjunction of energy accumulation mode, and high frequency output 
voltage, current channel ADC gain parameters, this is the condition of power calibration, and then power gain correction, next phase correction, phase correction was conducted after the complete power gain correction. All correction is carried out under the condition that the corresponding register parameter is zero.

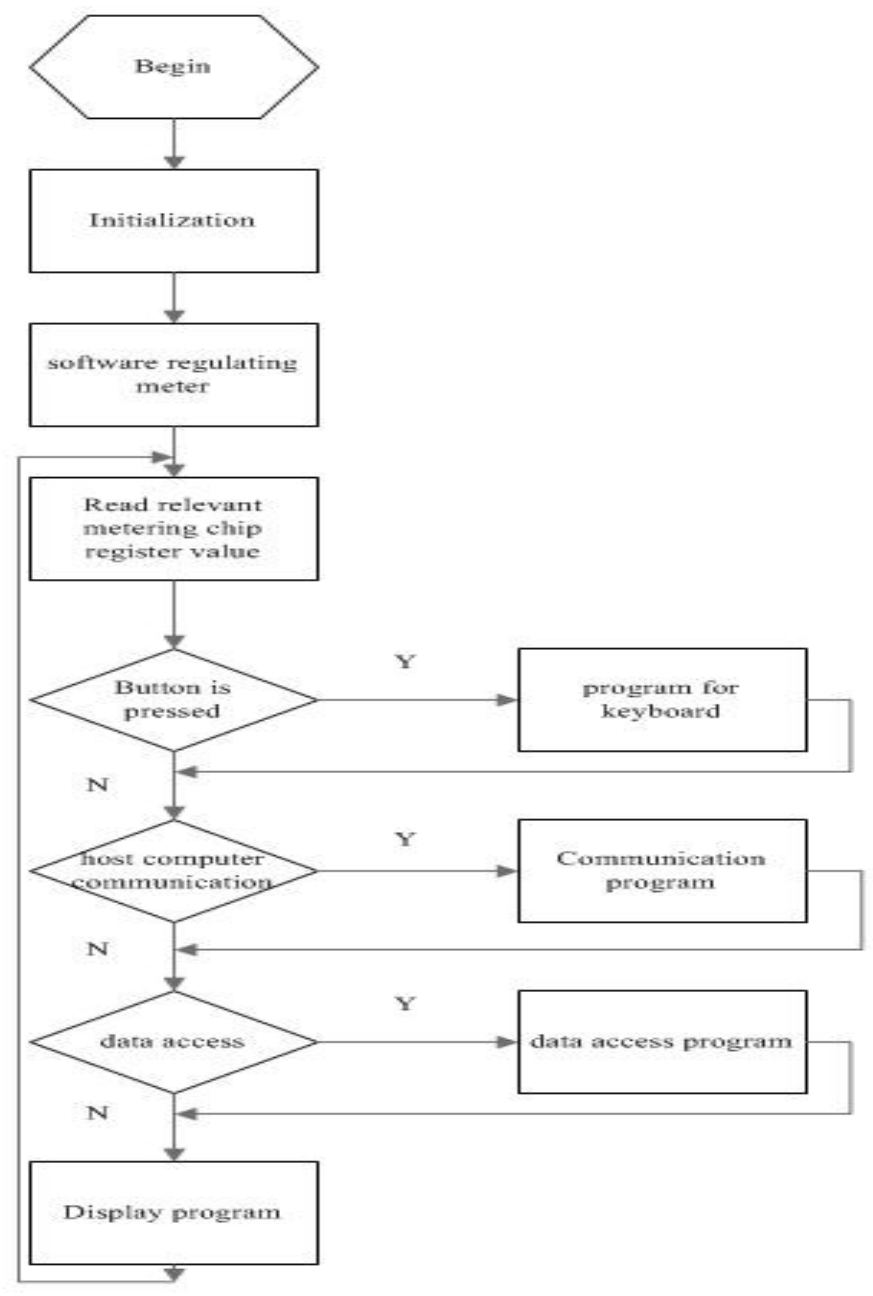

Fig. 4. Flow chart of system software

\section{Conclusions}

Using high performance CPU, STM32F103 as the control platform, with three-phase electric energy metering chip ATT7022E to realize the design of intelligent road light control system in the electric power parameter module, is able to measure the electrical parameters, with high integration, strong function, low cost, strong anti-interference ability, low power consumption, greatly reduce the calculation error and design complexity.

\section{References}

[1] Chao Yan, Jiancheng Ni: Software Guide, 2012,4,P.67-68(In Chinese).

[2] Xiaoyan Chen, Wei Gao, Huan Qin, Xiaodong Fang, Nana Gao: Electronic Measurement Technology, 2013,10,P.62-66(In Chinese).

[3] Xichun Liao, Jingzhe Ren, Zhigao Yang: Illuminating Engineering Journal, 2014,2,P.43-47(In Chinese).

[4] Junfeng Li, Feng Gao, Shaocai Jia: Science \& Technology Information, 2012,33,P.172-173(In Chinese).

[5] Aihua Yu, Riyang He: Journal of Zhejiang University of Science and Technology, 2008,3,P.175-178(In Chinese).

[6] Jiaoying Chen, Zhida Liang, Xiaocong Li: Hongshui River, 2012,5,P.71-74(In Chinese). 
[7] Pingchuan Zhang, Limin Hou: Journal of Xinyang Teachers College(Natural Science Edition), 2013,4,P.601-604(In Chinese). 